\title{
Solidification Front Shape of the Molten Metal in a Thermally Thin Cylinder
}

\author{
I. M. Tsun \\ Ural Federal University, ul. Mira 19, Yekaterinburg, 620002 Russia \\ e-mail:jossiftsoun@mail.ru \\ Received July 5, 2012
}

\begin{abstract}
Reported data are reviewed briefly. When a molten metal is extruded to produce a wire directly from the melt, a capillary stream $0.2-3 \mathrm{~mm}$ in diameter is directed into a cooling medium so that external heat exchange ensures faster solidification of the metal as compared to capillary disintegration of the stream into drops. The following two assumptions regarding the shape of the solidification front exist: a planar solidification front normal to the axis and a curved axisymmetric front surface. Both assumptions are considered. The assumption of a curved axisymmetric solidification front surface of the molten metal in a cylinder is shown to be more realistic.
\end{abstract}

DOI: $10.1134 / \mathrm{S} 0036029513020122$

\section{INTRODUCTION}

Active interest in solving solidification problems (Stefan problems) is related to the wide application of the industrial processes (especially in metallurgy) that use phase transformations accompanied by thermal effects to process materials. The majority of solutions have been obtained to date in studying the solidification of bulk metals (see, e.g., [1-7]). The problems of this type are characterized by large sizes, a large thermal mass, and the immobility of the bodies to be studied. These specific features make it possible to apply these solutions to investigating solidification in a moving thermally thin cylinder only from a methodological standpoint.

During the extrusion of a molten metal to produce wires directly from a melt, a capillary stream $0.2-$ $3 \mathrm{~mm}$ in diameter is directed in a cooling medium for external heat exchange to provide faster solidification of the metal as compared to capillary disintegration of the stream into drops. Some aspects of the flow and disintegration of a capillary stream and various types of external heat exchange in this technological process were analyzed (see, e.g., [8-17]). In this work, we consider some features of the mathematical simulation of the solidification front motion in a solidifying stream.

Ostroumov $[18,19]$ studied the problem of solidification of an infinite liquid cylinder pulled at a constant speed from a furnace, and his results are important for the problems of extrusion of metallic melts. However, some initial assumptions (variable solidification temperature, initial supercooling of a liquid, initial setting of a planar solidification front normal to the axis) restrict the application of these investigations.

\section{ANALYSIS OF THE EXISTING SOLUTION}

The cycle of studies performed by Berman et al. $[20,21]$ is of particular interest from the standpoint of investigating the process under study due to the development and improvement of the processes of production of a cast microwire in glass insulation 5-30 $\mu \mathrm{m}$ in diameter at a speed up to $8 \mathrm{~m} / \mathrm{s}$. Those authors analyzed the problem of solidification of a moving liquid cylinder that is not thermally isolated from the environment under the following boundary conditions. (The formulation of the problem and its solution are modified by us with allowance for our problem.)

The initial cylinder temperature is

$$
\vartheta(0)=\vartheta_{\text {ini }}>\vartheta_{\mathrm{m}},
$$

where $\vartheta=t-t_{\text {at }}$ and $t_{\mathrm{m}}$ is the melting (solidification) temperature. Ambient temperature $t_{\text {at }}$ is constant and is taken to be a reference point. The solidification front, i.e., plane $z=l$ ( $z$ is the axial coordinate), divides the cylinder into two parts, namely, liquid (subscript 1) and solid (subscript 2) parts. The thermophysical parameters are averaged and are considered to be the same in both parts. At the isothermal interface, we have

$$
\begin{gathered}
\vartheta_{1}(l)=\vartheta_{2}(l)=\vartheta_{\mathrm{m}}, \\
\lambda \frac{d \vartheta_{1}}{d z}-\lambda \frac{d \vartheta_{2}}{d z}=\rho v \Delta H_{\mathrm{m}},
\end{gathered}
$$

where $v$ is the cylinder speed, $\Delta H_{\mathrm{m}}$ is the melting (solidification) heat, $\lambda$ is the thermal conductivity, $\rho$ is the metal density, and

$$
\left.\vartheta_{2}\right|_{z \rightarrow \infty} \rightarrow 0 .
$$


Berman derived a dynamic heat conduction equation for a moving cylinder that takes into account heat transfer by both heat conduction and the cylinder motion,

$$
\frac{d^{2} \vartheta_{i}}{d z^{2}}-\frac{\underline{v}}{a} \frac{d \vartheta_{i}}{d z}-S^{2} \vartheta_{i}=0
$$

where

$$
S^{2}=4 \alpha /(\lambda d)
$$

$a$ is the thermal diffusivity, $\alpha$ is the heat-transfer coefficient of the surface, $i=1$ and 2 , and $d$ is the cylinder diameter.

We should determine quasi-stationary temperature fields $\vartheta_{1}(z)$ and $\vartheta_{2}(z)$ and the position of boundary $z=l$.

The value of $l$ is found using boundary condition (2) from the equation

$$
\left.\vartheta_{1}(z)\right|_{z=l}=\left.\vartheta_{2}(z)\right|_{z=l}=\vartheta_{\mathrm{m}} .
$$

Here, $\vartheta_{1}(z)$ and $\vartheta_{2}(z)$ are the found temperature fields

$$
\begin{gathered}
\vartheta_{1}=\frac{\rho \cup \Delta H_{\mathrm{m}}}{2 \lambda g} \frac{\exp (k z)-\exp (\varepsilon z)}{\exp (k l)}+\vartheta_{\mathrm{ini}} \exp (\varepsilon z), \\
\vartheta_{2}=\frac{\rho \cup \Delta H_{\mathrm{m}}}{2 \lambda g} \frac{\exp (k l)-\exp (\varepsilon l)}{\exp (k l)} \exp (\varepsilon(z-l)) \\
\quad+\vartheta_{\mathrm{ini}} \exp (\varepsilon z), \\
g=\left(\frac{\mathrm{v}^{2}}{4 a^{2}}+S^{2}\right)^{1 / 2}, \quad k=\frac{v}{2 a}+g, \quad \varepsilon=\frac{v}{2 a}-g .
\end{gathered}
$$

Equation (6) has roots in the region $l>0$ under the necessary condition

$$
\vartheta_{\mathrm{m}}>\Delta H / c
$$

where $c$ is the heat capacity of the metal.

A supercooling forms before the solidification front in the liquid part of the cylinder at the pulling speed

$$
v>v_{\mathrm{cr}}=a S \sqrt{\frac{c \vartheta_{\mathrm{m}}}{\Delta H_{\mathrm{m}}\left(1+\frac{\Delta H_{\mathrm{m}}}{c \vartheta_{\mathrm{m}}}\right)}} .
$$

Minimum temperature $\vartheta_{\text {min }}<\vartheta_{\mathrm{m}}$ is reached at point $z_{\min } \in(0, l)$. The dependence $\vartheta_{\min }=\vartheta_{\text {min }}(d, v)$ has an asymptotic decreasing character when $d$ or $v$ increases. The limiting possible temperature of the supercooled liquid is

$$
\vartheta_{\text {min lim }}=\vartheta_{\mathrm{m}}-\Delta H_{\mathrm{m}} / c \text {. }
$$

We introduce the maximum possible supercooling depth

$$
\vartheta_{\mathrm{d}}=\vartheta_{\mathrm{m}}-\vartheta_{\min \lim }=\Delta H_{\mathrm{m}} / \mathrm{c} \text {. }
$$

For copper, we have $\vartheta_{\mathrm{d}}=567^{\circ} \mathrm{C}$ [21]; for steel (iron), we have $\vartheta_{\mathrm{d}}=325^{\circ} \mathrm{C}$ [22].

The experimental data in [21] indicate that the assumption of a planar solidification front is justified at a diameter $d=5-30 \mu \mathrm{m}$ and a speed $v=1-8 \mathrm{~m} / \mathrm{s}$. It is interesting to estimate its correctness at the parameters important for the extrusion of molten metals, namely, $d=200-300 \mu \mathrm{m}$ and $\mathrm{v}=1-3 \mathrm{~m} / \mathrm{s}$.

We now modify Eq. (8). We express $\vartheta_{\text {ini }}$ in terms of $\vartheta_{\mathrm{m}}$ using Eq. (7) and boundary condition (2), substitute this expression into Eq. (8), and obtain

$$
\vartheta_{2}(z)=\vartheta_{\mathrm{m}} \exp (\varepsilon(z-l)),
$$

where

$$
\begin{aligned}
& \varepsilon=-\frac{v}{2 a}\left[\left(1+\frac{4 a^{2} S^{2}}{v^{2}}\right)^{1 / 2}-1\right] \\
& =-\frac{v}{2 a}\left[\left(1+\frac{16 \alpha a}{c \rho d v^{2}}\right)^{1 / 2}-1\right],
\end{aligned}
$$

from Eqs. (9) and (5a).

If the origin of the axial coordinate is translated to the solidification front to analyze the temperature field of the solidified part of the cylinder, we have $z^{\prime}=$ $z-l$ and

$$
\vartheta_{2}(z)=\vartheta_{\mathrm{m}} \exp \left(\varepsilon z^{\prime}\right)
$$

Therefore, the cooling of the solid part of the cylinder can be represented as the cooling of a moving single-phase cylinder from the initial temperature $\left(\vartheta_{\mathrm{m}}\right)$; is independent of the initial temperature of the liquid cylinder $\vartheta_{\text {ini }}$ and the heat of solidification $\Delta H_{\mathrm{m}}$; and is determined by the temperature at the head end $\left(\vartheta_{\mathrm{m}}\right)$, speed $v$, diameter $d$, heat exchange with the environment $\alpha$, and the physical parameters of the solid cylin$\operatorname{der}(\rho, c, a)$.

According to [21], $4 a^{2} S^{2} / \mathrm{v}^{2}$ is on the order of $10^{-2}$ at $d=5-30 \mu \mathrm{m}$. At the values of diameter $d$ under study, this quantity is on the order of $10^{-3}-10^{-4}$; therefore, we can use the transformation

$\varepsilon=-\frac{v}{2 a}\left[\left(1+\frac{4 a^{2} S^{2}}{v^{2}}\right)^{1 / 2}-1\right]=-\frac{v}{2 a} \frac{2 a^{2} S^{2}}{v^{2}}=-\frac{a S^{2}}{v}$.

Taking into account Eq. (5a) for $S^{2}$, we have

$$
\varepsilon=-\frac{4 \alpha}{c \rho d v}
$$

and Eq. (16) takes the form

$$
\vartheta_{2}(z)=\vartheta_{\mathrm{m}} \exp \left(-\frac{4 \alpha}{c \rho d v} z^{\prime}\right) .
$$

It is known [23] that the temperature conditions of a thermally thin cylinder is described by the equation

$$
\frac{\vartheta}{\vartheta_{\text {ini }}}=\exp (-2 \text { Bi Fo })=\exp \left(-\frac{4 \alpha}{c \rho d} \tau\right),
$$




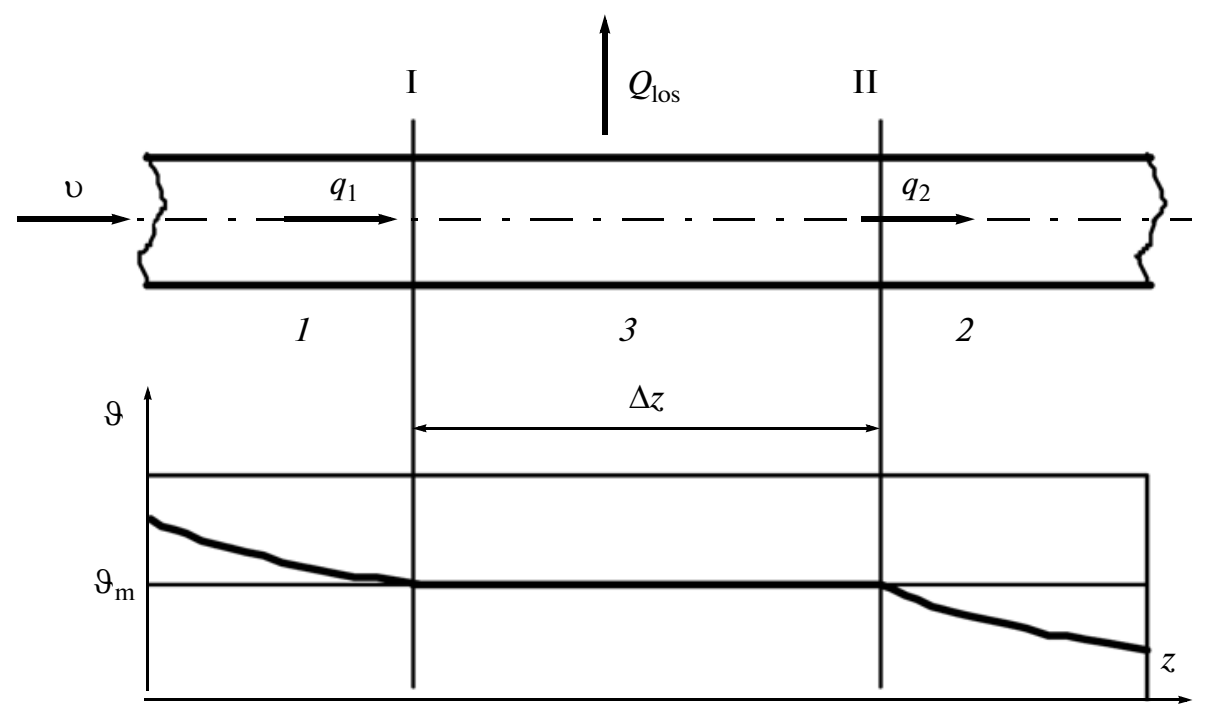

Fig. 1. Schematic diagram for estimating the solidification region length in a cylinder.

where $\vartheta$ and $\vartheta_{\text {ini }}$ are the current and initial cylinder temperatures, respectively,

$$
\mathrm{Bi}=\frac{\alpha d}{2 \lambda}, \mathrm{Fo}=\frac{4 a \tau}{d^{2}} .
$$

As a result of the substitution $\tau=z / v$, we obtain

$$
\vartheta=\vartheta_{\text {ini }} \exp \left(-\frac{4 \alpha}{c \rho d v} z\right)
$$

A comparison of Eqs. (18) and (19) shows that the temperature conditions of the solidified part of the cylinder described by Eq. (18) are identical to the wellknown cooling conditions of a cylinder without phase transformations (Eq. (19)); therefore, the heat generation in the solidification front weakly affects the temperature field of the solidified part of the cylinder $\vartheta_{2}(z)$. Then, the problem of the way of heat removal from a planar solidification front normal to the axis and the assumption of this solidification front shape are to be refined.

We now estimate the limits of application of the assumption of a planar solidification front in a cylinder. Let solidification take place in a cylinder segment of length $\Delta z$ (Fig. 1).

The thermal balance of the solidification section is

$$
\begin{aligned}
& F\left(q_{1}+c_{1} \rho \cup \vartheta_{\mathrm{m}}\right)+F \cup \rho \Delta H_{\mathrm{m}} \\
= & F\left(q_{2}+c_{2} \rho \cup \vartheta_{\mathrm{m}}\right)+\alpha \Pi \vartheta_{\mathrm{m}} \Delta z .
\end{aligned}
$$

Here, $F$ and $\Pi$ are the cross-sectional area and perimeter, respectively; $F\left(q_{1}+c_{1} \rho \cup \vartheta_{\mathrm{m}}\right)$ is the heat introduced through section I; $F\left(q_{2}+c_{2} \rho v \vartheta_{\mathrm{m}}\right)$ is the heat introduced through section II; $F \cup \rho \Delta H_{\mathrm{m}}$ is the heat released due to solidification; $\alpha \Pi \vartheta_{\mathrm{m}} \Delta z$ is loss $Q_{\text {los }}$ to the environment; and $q_{1}$ and $q_{2}$ are the specific heat flows by heat conduction through sections I and II, respectively.

It follows from equality (20) that

$$
\frac{\Delta z}{d}=\frac{1}{4 \alpha \vartheta_{\mathrm{m}}}\left[\left(q_{1}-q_{2}\right)+\left(c_{1}-c_{2}\right) \rho v \vartheta_{\mathrm{m}}+v \rho \Delta H_{\mathrm{m}}\right] \text {. }
$$

The assumption of a planar solidification front normal to the axis means that $\Delta z=0$; then, it follows from Eq. (21) that

$$
\left(q_{1}-q_{2}\right)+\left(c_{1}-c_{2}\right) \rho v \vartheta_{\mathrm{m}}+v \rho \Delta H_{\mathrm{m}}=0 .
$$

When averaging and equating the heat capacities of the liquid and solid phases, we obtain

$$
\left(q_{1}-q_{2}\right)+v \rho \Delta H_{\mathrm{m}}=0,
$$

which coincides with the meaning of boundary condition (3).

Using Eq. (14), we determine heat flow $q_{2}$,

$$
q_{2}=-\left.\lambda \frac{d \vartheta_{2}}{d z}\right|_{z=l}=-\lambda \vartheta_{\mathrm{m}} \varepsilon
$$

or, with allowance for Eq. (17), we have

$$
q_{2}=\lambda \vartheta_{\mathrm{m}} \frac{a S^{2}}{\mathrm{v}}
$$

It follows from equality (23) that

$$
q_{1}=q_{2}-v \rho \Delta H_{\mathrm{m}}=\lambda \vartheta_{\mathrm{m}} \frac{a S^{2}}{\mathrm{v}}-v \rho \Delta H_{\mathrm{m}} .
$$

Thus, when speed $v$ increases, heat flow $q_{2}$ by heat conduction to the solidified part decreases. At sufficiently high speeds $v$ of pulling a liquid cylinder, a regime at which $q_{1}<0$ takes place (see equality (25)), which means the necessity of a supercooled liquid before solidification. 
It is known $[13,24]$ that the supercooling of a melt during metal solidification is related to thermodynamic and kinetic specific features. Here, this supercooling is necessary to satisfy one of the problem assumptions, namely, the assumption of a planar solidification front $(\Delta z=0)$.

Large volumes in most metals can be supercooled only insignificantly (by about $1{ }^{\circ} \mathrm{C}$ [24]) before solidification. However, "if the metal volume is divided into numerous small droplets, many of them can be strongly supercooled" [24, p. 77]. In particular, the experimentally achieved supercooling is $236^{\circ} \mathrm{C}$ for copper and $295^{\circ} \mathrm{C}$ for iron. According to [25], the supercooling of rapidly cooled iron droplets $30-50 \mu \mathrm{m}$ in diameter is $300-350^{\circ} \mathrm{C}$. As noted above, the supercooling depth required for a planar solidification front does not exceed $567^{\circ} \mathrm{C}$ for copper and $325^{\circ} \mathrm{C}$ for iron. For small diameters $(5-30 \mu \mathrm{m}$ or smaller), the supercooling required according to the problem assumption and the thermodynamically achieved supercooling are likely to have the same order of magnitude. Therefore, the assumption of a planar solidification front is formally fulfilled in these cases under the real conditions of production of a cast microwire in glass insulation.

The values at which $q_{1}<0$ are determined from the inequality

$$
q_{2}-v \rho \Delta H_{\mathrm{m}}<0 .
$$

which is a consequence of equality (25). We substitute the expression for $\varepsilon$ according to Eq. (9) into Eq. (24), take into account Eq. (26), and eventually obtain an inequality coinciding with Eq. (11).

For solidification condition (11) to exist in the liquid part of the cylinder, the preliminary supercooling should have a certain depth to meet the requirement $q_{1}<0$. If we make a more general assumption of a curved solidification front, it is seen from Eq. (21) that both heat flows $q_{1}$ and $q_{2}$ at $\Delta z>0$ can be positive due to the fact that the heat from a solidification region in this case is removed directly to the environment. A curved solidification front shape during melt extrusion is also indicated by experimental data. For example, Kavesh [8] used metallography to determine the direction of the normal to the solidification front in a zinc cast wire $267 \mu \mathrm{m}$ in diameter prepared by melt extrusion into water. The angle between the normal and the wire axis near the surface was $79^{\circ}$. If the solidification front normal to the axis was planar in this case, this angle would be $0^{\circ}$.

\section{DERIVATION AND ANALYSIS \\ OF AN EQUATION FOR A CURVED AXISYMMETRIC SHAPE OF THE SOLIDIFICATION FRONT}

An analysis of the boundary conditions and limitations of extrusion shows that, to a first approximation, we can restrict ourselves to the consideration of a mathematical model in which a thermally thin stream (cylinder) of an infinite length has the solidification temperature of the molten metal at the entrance into the infinite volume of a cooling medium.

Let a disk of thickness $\Delta l$ travel a distance $\Delta z=v \Delta \tau$ along with the stream in the motion direction. In this case, the disk releases a certain quantity of heat into the environment as a result of the solidification of a ring of thickness $\Delta r$ in the disk,

$$
\Delta Q=-\Delta H_{\mathrm{m}} 2 \pi r \Delta r \Delta l \rho_{1} .
$$

The heat losses are

$$
\Delta Q=q 2 \pi r_{\text {out }} \Delta l \Delta \tau .
$$

Here, $q$ is the specific heat flow from the outer surface; $r$ is the current radius of the solidification front; $r_{\text {out }}$ is the outside radius of the solidifying stream,

$$
r_{\text {out }}=r_{1} \sqrt{\left(r / r_{1}\right)^{2}+\left[1-\left(r / r_{1}\right)^{2}\right]\left(\rho_{1} / \rho_{\mathrm{s}}\right)} ;
$$

$r_{1}$ is the initial stream radius; $\rho_{1}$ and $\rho_{\mathrm{s}}$ are the metal densities in the liquid and solid state, respectively; and $\Delta H_{\mathrm{m}}$ is the melting heat.

We set Eq. (27) equal to Eq. (28), pass to the limit at $\Delta \tau \rightarrow 0$, designate

$$
\left(r / r_{1}\right)=R,\left(\rho_{1} / \rho_{\mathrm{s}}\right)=\Omega, r_{1}=d_{1} / 2,
$$

and obtain an equation that describes the solidification front motion over the cross section of the stream,

$$
\frac{d R}{d \tau}=-\frac{2 q}{\rho_{1} \Delta H_{\mathrm{m}} d_{1}} \sqrt{1+\frac{1-R^{2}}{R^{2}} \Omega .}
$$

As a result of the substitution

$$
\frac{d R}{d \tau}=\frac{d R}{d z} \frac{d z}{d \tau}=v \frac{d R}{d z}
$$

we obtain the following differential equation of the solidification front:

$$
\frac{d R}{d z}=-\frac{2 q}{v \rho_{1} \Delta H_{\mathrm{m}} d_{1}} \sqrt{1+\frac{1-R^{2}}{R^{2}} \Omega,}
$$

The solution to this equation along with the condition

$$
\left.R\right|_{z=0}=1
$$

specifies the surface shape forming the solidification front,

$$
Z_{d}=2\left[1-\sqrt{R^{2}(1-\Omega)+\Omega}\right] /\left[N_{q}(1-\Omega)\right],
$$

where

$$
Z_{d}=z / d_{1}, N_{q}=4 q /\left(v \rho_{1} \Delta H_{\mathrm{m}}\right) .
$$

Complex $N_{q}$ characterizes the ratio of the specific heat flow from the outer surface to the heat flow along the axis created by the moving solidifying stream. 
The outer surface radius of the stream during solidification is determined according to Eq. (29) from the equality

$$
R_{\text {out }}=r_{\text {out }} / r_{1}=\sqrt{R^{2}(1-\Omega)+\Omega} .
$$

Combining Eqs. (32) and (33), we obtain the following equation for the outer surface of the solidifying stream:

$$
R_{\text {out }}=1-N_{q}(1-\Omega) Z_{d} / 2 .
$$

Thus, the stream in the solidification region has the shape of a truncated cone due to the decrease in the metal volume during solidification.

The solidification region length is

$$
L_{\text {sol }}=l_{\text {sol }} / d_{1}=\left.Z_{d}\right|_{R=0}=2 /\left[N_{q}\left(1+\Omega^{0.5}\right)\right] \text {. }
$$

The final radius of the solidified stream is

$$
R_{\text {out } 2}=d_{2} / d_{1}=\left.R_{\text {out }}\right|_{R=0}=\Omega^{0.5} .
$$

It is known [21] that the volume change during solidification $\Delta V / V_{1}$ is a relatively small quantity: for iron, $\Delta V / V_{1}=-2.2 \times 10^{-2}$; for copper, $\Delta V / V_{1}=-4.1 \times$ $10^{-2}$; and for aluminum, $\Delta V / V_{1}=-6.0 \times 10^{-2}$. We can show that $\beta=(1-\Omega)=-\left(\Delta V / V_{1}\right)$; therefore, we have $\beta \leq 6 \times 10^{-2}$. Under this condition, Eq. (32) can be reduced to the approximate form

$$
Z_{d}^{\mathrm{app}}=\frac{2}{N_{q}\left(1+\Omega^{0.5}\right)}\left(1-R^{2} \frac{1+\Omega^{0.5}}{2 \Omega^{0.5}}\right) .
$$

The accuracy of $\delta$ calculations by Eq. (37) is determined by the relationships

$$
Z_{d}^{\mathrm{app}}>Z_{d},\left.\left(Z_{d}^{\mathrm{app}}-Z_{d}\right)\right|_{\beta \leq \delta} \leq \delta / 4 .
$$

For the case $\rho_{1}=\rho_{\mathrm{s}}$ (i.e., $\Omega=1, \beta=0$ ), it follows from Eq. (5) or (6) that

$$
Z_{d}=\left(1-R^{2}\right) / N_{q} .
$$

Therefore, the solidification front has a shape close to a paraboloid of revolution; the smaller the change in the density during solidification, the closer the front to this shape.

The increase in the size along the axial coordinate induced by the change in the density during solidification at any $R=$ const can be taken into account using Eqs. (32) and (38) and the expression

$$
\frac{\Delta Z_{d}}{\left.Z_{d}\right|_{\beta=0}}=\frac{\left.Z_{d}\right|_{\beta \neq 0}-\left.Z_{d}\right|_{\beta=0}}{\left.Z_{d}\right|_{\beta=0}}=\frac{\beta\left(1-R^{2}\right)}{4} .
$$

In particular, for iron at $\beta=2.2 \%$, the elongation of solidification region $L_{\text {sol }}$ is $\sim 0.5 \%$.

In a first approximation, solidification region length $L_{\text {sol }}^{\text {app }}$ can be estimated from Eq. (35) in the form

$$
L_{\mathrm{sol}}^{\mathrm{app}}=\left(1+\frac{1}{4} \beta\right) / N_{q} .
$$
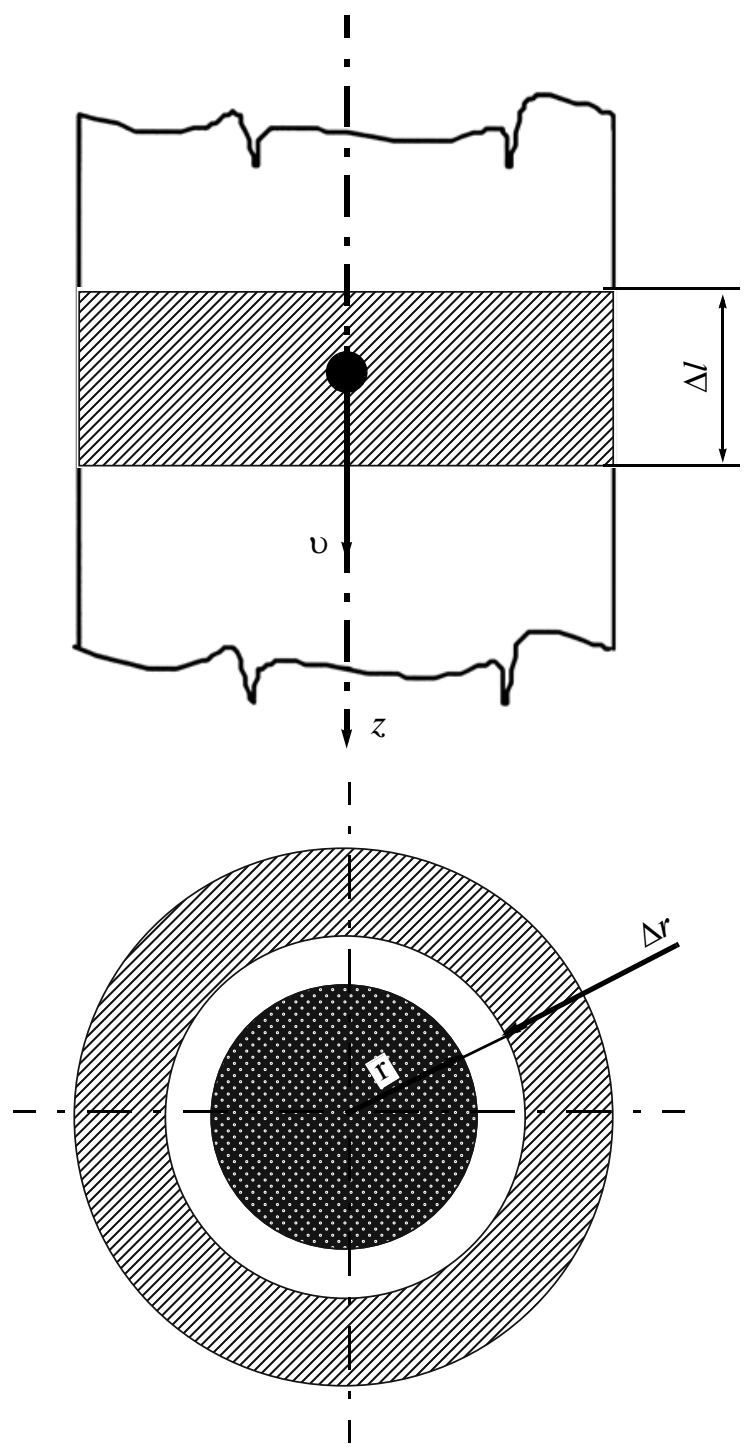

Fig. 2. Schematic diagram for deriving the solidification front equation.

Therefore, the solidification region length is determined by the value of complex $N_{q}$. Allowing for the limits of the related variables, we obtain

$$
N_{q} \sim\left(10^{-2}-10^{-5}\right) \text {. }
$$

Correspondingly, we have $L_{\text {sol }} \sim 10^{2}-10^{5}$. Thus, the solidification region length along the axis is 100100000 diameters of a cylinder $0.5-3 \mathrm{~mm}$ in diameter, and the assumption of a planar solidification front normal to the solidification axis is seen to be far from reality.

The only quantity in complex $N_{q}$ that can be controlled over a wide range is the specific heat flow from the outer surface $q$. Hence, the intensification of external heat exchange is the only thermophysical factor to decrease the solidification region to an acceptable length, which sets the problem of a mathematical 
simulation of this factor under the specific conditions of extrusion of a molten metal.

\section{CONCLUSIONS}

Our analysis of two mutually excluding assumptions of the solidification front shape in a thermally thin cylinder during mathematical simulation shows that the assumption of a curved axisymmetric solidification front yields more realistic results.

\section{REFERENCES}

1. B. Ya. Lyubov, Theory of Solidification in Large Volumes (Nauka, Moscow, 1985).

2. V. A. Zhuravlev and E. M. Kitaev, Thermophysics of the Formation of a Continuous Ingot (Metallurgiya, Moscow, 1974).

3. Yu. A. Samoilovich, "Dynamics of nonequilibrium alloy solidification," Fiz. Khim. Obrab. Mater., No. 3, 85-92 (1978).

4. Yu. A. Samoilovich, "Heat exchange between a melt stream and the solidification front in an ingot," Fiz. Khim. Obrab. Mater., No. 6, 28-35 (1978).

5. N. A. Spirin, V. S. Shvydkii, V. I. Lobanov, and V. V. Lavrov, Introduction to the System Analysis of the Thermophysical Processes in Metallurgy (UGTU-UPI, Yekaterinburg, 1999).

6. V. S. Shvydkii, N. A. Spirin, Yu. G. Yaroshenko, et al., System Theory Elements and Numerical Methods for Simulating Heat-and-Mass Transfer Processes (Intermet Inzhiniring, Moscow, 1999).

7. A. A. Samarskii and P. N. Vabishchevich, Computational Heat Transfer (Editorial URSS, Moscow, 2003).

8. Sh. Kavesh, "Melt spinning of metal fibers" American Institute of Chemical Engineers, Symposium Series 74 (180), 1-15 (1978).

9. Sh. Kavesh, "Liquid quenching of free jet spun metal filaments," US Patent 3845805.

10. G. K. Subbotin and I. M. Tsun, "Radiative heat transfer between an isothermal surface and a long thermally thin body," in Thermophysics of Melting Steels and Alloys (UPI, Sverdlovsk, 1976), Vol. 4, pp. 84-89.

11. G. K. Subbotin, I. M. Tsun, V. P. Petyshin, and V. I. Luk'yanov, "Specific features of the heat transfer during the extrusion of molten metals to produce a cast wire," in Thermophysics of Melting Steels and Alloys (UPI, Sverdlovsk, 1977), Vol. 5, pp. 31-37.
12. E. V. Toropov, G. K. Subbotin, and I. M. Tsun, "Boiling of liquid on the surface of a solidifying molten metal stream," in Boiling and Condensation (Rizhsk. Politekhn. Inst., Riga, 1979), pp. 35-39.

13. I. M. Tsun, "Motion of an infinite cylinder along the axis downward in a viscous dense medium," in Fundamental and Applied Studies: Transactions of Lecturers and Postgraduate Students of the Physicomathematical Department of the Magnitogorsk State Pedagogical Institute (MGPI, Magnitogorsk, 1998), pp. 89-95.

14. I. M. Tsun, "Solution of the boundary-value problem of the longitudinal Couette flow between cylindrical surfaces," in Problems of Mathematical Education in Pedagogical Institutes at the Modern Stage (ChGPU, Chelyabinsk, 1998), p. 36.

15. I. M. Tsun, "Analytical aspects of the problem of appearance of a vapor phase in a boundary layer," in Proceedings of All-Russia Conference on Problems of Physical-Mathematical Education in Pedagogical Institutes of Russia at the Modern Stage (MGPI, Magnitogorsk, 1999), pp. 94-95.

16. I. M. Tsun, "Experimental investigations of the stability of capillary jets," Vestn. MaGU, nos. 2-3, 225-232 (2001).

17. I. M. Tsun, "Mathematical models of solidification in a cylinder," Vestn. Tambovskogo Univ., Ser. Estestvennye i Tekhnicheskie Nauki 12 (4), 552-554 (2007).

18. G. A. Ostroumov, Zh. Tekh. Fiz. 25 (10) (1955).

19. G. A. Ostroumov, Zh. Tekh. Fiz. 29 (2), 239-246 (1959).

20. N. R. Berman, "On the theory of solidification of a thin moving rod (dynamic Stefan problem)," Extended Abstract of Cand. Sci. (Phys.-Math.) Dissertation, Moscow, 1968.

21. E. Ya. Badinter, N. R. Berman, N. F. Drabenko, et al., Cast Microwire and Its Properties (Shtiintsa, Kishinev, 1973).

22. G. N. Oiks and Kh. M. Ioffe, Steelmaking. Calculations (Metallurgiya, Moscow, 1975).

23. Thermotechnical Handbook (Energiya, Moscow, 1976).

24. B. Chalmers, Principles of Solidification (Wiley, New York, 1964).

25. V. P. Kostyuchenko and D. E. Ovsienko, in Mechanism and Kinetics of Solidification (Nauka i Tehnika, Minsk, 1964).

Translated by K. Shakhlevich 